\title{
Nuevos modelos en el aprendizaje de la radiodifu- sión para el Espacio Europeo de Educación Superior
}

\author{
Yolanda BERDASCO GANCEDO \\ Universidad a Distancia de Madrid (UDIMA) \\ yolanda.berdasco@udima.es \\ Ana Virginia RUBIO JoRDÁN \\ Universidad a Distancia de Madrid (UDIMA) \\ anavirginia.rubio@udima.es
}

Recibido: $25 / 10 / 2012$

Aceptado: 23/01/2013

\begin{abstract}
Resumen Superior (EEES), Enseñanza a distancia, TIC's.

\section{New Learning Models of Broadcasting for the European Higher Education Area}

La radio como disciplina está presente desde sus comienzos en los planes de estudio de las facultades de Ciencias de la Información de las universidades españolas. Por su propia idiosincrasia, la radiodifusión requiere de una enseñanza eminentemente práctica, además de teórica, muy en consonancia con el espíritu que transmite el Espacio Europeo de Educación Superior (EEES) y en respuesta a las demandas que reclama la sociedad actual y el mundo profesional periodístico de hoy. El objetivo de este trabajo es poner de manifiesto las posibilidades que ofrece la educación a distancia a la hora de afrontar asignaturas como Periodismo Radiofónico, en el que los estudiantes han tenido que realizar una serie de actividades en grupo destinadas a simular el trabajo que se realiza en la redacción de una emisora de radio.

Palabras clave: Enseñanza superior de la radio, Periodismo radiofónico, Espacio Europeo de Educación

\begin{abstract}
As a theoretical discipline, radio has been present in the curricula of schools of communication and journalism of Spanish universities since their first steps. Apart from this traditional and theoretical frame and due to its own special features, radio broadcasting requires a highly practical teaching in keeping with the European Higher Education Area (EHEA) spirit. This practical approach offers also a response to society demands and to our journalistic professional environment.

The main aim of this paper is showing the potential of distance education to deal with subjects such as radio journalism, in which students had to perform a series of group activities aimed at simulating daily tasks and routines in a radio station.

Keywords: Higher Education Radio, Radio Journalism, European Higher Education Area (EHEA), e-learning, ICT's.

\section{Referencia normalizada}

BERDASCO GANCEDO, Yolanda y RUBIO JORDÁN, Ana Virginia: (2013): "Nuevos modelos en el aprendizaje de la radiodifusión para el Espacio Europeo de Educación Superior". Estudios sobre el mensaje periodístico. Vol. 19. Núm. especial abril, págs.: 605-612. Madrid, Servicio de Publicaciones de la Universidad Complutense.
\end{abstract}

Sumario: 1. Introducción. 2. Metodología. 3. Desarrollo; 3.1. Valoración de las herramientas metodológicas empleadas por parte del alumnado. 4. Conclusiones. 5. Referencias bibliográficas.

\section{Introducción}

En los últimos años la llegada de Internet y la aparición de las nuevas tecnologías han creado una cultura multimedia en torno a lo audiovisual que ha transformado el modo 
en el que el ser humano accede a la información. La radio ha sido tradicionalmente un área a la que los planes de estudio de las universidades españolas han prestado una atención menor en favor del periodismo impreso, con una reducción de la carga lectiva y con asignaturas basadas en la teoría y alejadas de la práctica profesional propia de la actividad que desarrollará un periodista de radio en su vida diaria. Los contenidos audiovisuales se han decantado, en su mayor parte, por la imagen y la didáctica de la televisión, como medio predominante, en detrimento del medio radiofónico. A pesar de ello y de la aparición de nuevos medios, la radio ha demostrado mantenerse como una referencia para la comunicación. "La radio es una superviviente por naturaleza, no olvidemos que la base de la radio está marcada por la misma esencia de la comunicación, y ésta, por su naturaleza, también forma parte de nuestro instinto de supervivencia" (Tenorio, 2008: 17).

La adaptación de los estudios de Periodismo al Plan Bolonia se remonta en nuestro país a la Ley Orgánica 4/2007 de 12 de abril. En el Libro Blanco de las Titulaciones de Comunicación, publicado por ANECA en 2005, destacan cuatro perfiles profesionales para el grado en Periodismo, teniendo cabida, en el primero de ellos, los medios audiovisuales: "Redactor/a de información periodística en cualquier tipo de soporte. [...] Comprende las tareas de redactor, reportero, presentador y director de uno o de varios de ellos, incluido el diseño escritura y ejecución de guiones de reportajes y documentales audiovisuales o multimedia".

Por su propia idiosincrasia, la enseñanza de la radiodifusión requiere de una metodología fundamentalmente práctica muy en la línea de lo que promueve el Espacio Europeo de Educación Superior (EEES).

Desde que comenzara a impartir los estudios de Periodismo durante el curso académico 2009/2010, la Universidad a Distancia de Madrid (UDIMA) ha realizado una firme apuesta por la incorporación de las nuevas tecnologías frente a los modelos de enseñanza tradicionales.

Los objetivos principales del título son los de satisfacer la demanda de profesionales formados en el deber moral y ético, los conocimientos teóricos necesarios, así como el dominio tecnológico y el ejercicio de la práctica profesional en el ámbito de la comunicación, tan demandado hoy en día por las empresas periodísticas.

Las materias que forman parte del grado y que tienen que ver con la radiodifusión se denominan Comunicación Radiofónica y Periodismo Radiofónico. Ambas asignaturas son de tercer curso, tienen carácter obligatorio y una carga lectiva de 6 créditos ECTS, cada una de ellas.

En el presente curso académico 2012/2013, los estudiantes que comenzaron sus estudios de Periodismo en UDIMA se disponen a cursar el último año del Grado. Las asignaturas que tratan sobre el medio radiofónico se encuentran, como hemos comentado, en tercer curso, con lo cual, los alumnos ya se han enfrentado a la experiencia que supone aprender radio a distancia y podemos, por tanto, realizar un estudio para extraer unas primeras conclusiones sobre los resultados en base a las experiencias formativas aplicadas. 


\section{Metodología}

Como defienden muchos estudiosos del medio, la didáctica de las asignaturas de radio debe contemplar la necesidad de estimular a los futuros profesionales de radio y de realizar prácticas eficaces en pequeños grupos. "Cualquier actividad radiofónica de producción ha de desarrollarse en pequeños grupos. Mi experiencia indica que lo ideal es constituir equipos de seis personas (que pueden trabajar individualmente, por parejas, tríos o en conjunto) y no sobrepasar nunca el número de diez. Importa mucho que el profesor sepa incentivar y orientar el trabajo en equipo, de modo que los alumnos ejerciten también funciones de liderazgo y aprendan a cooperar en la consecución de objetivos comunes" (Merayo, 2010: www.bocc.ubi.pt/pag/merayo-arturo-didacticacomunication-radiofonica.pdf).

Con estas premisas en mente, diseñamos las asignaturas para poder cumplir estos objetivos, salvando los obstáculos y permitiendo a nuestros estudiantes llevar a cabo unos ejercicios prácticos que simulan la realidad que se vive en una redacción de un medio radiofónico.

Con este objetivo, nos decantamos por un modelo que nos permitiera realizar el trabajo en grupo y obtener como resultado final, un espacio radiofónico de características similares a los que se emiten a diario en las emisoras de nuestro país. En UDIMA el módulo de Información Radiofónica cuenta con 12 créditos de materias obligatorias, distribuidas en el primer y segundo semestre del tercer curso del grado en Periodismo y que son Comunicación Radiofónica y Periodismo Radiofónico.

En la primera parte del módulo, la que corresponde a Comunicación Radiofónica, tratamos de sentar las bases y de proporcionar al estudiante las habilidades y los conocimientos básicos para poder dar los primeros pasos en el mundo de la radio, mientras que en Periodismo Radiofónico nos centramos en la práctica de la materia.

La herramienta principal es el aula virtual que descansa sobre la plataforma Moodle y que nos sirve de lugar de encuentro entre los alumnos y el profesor; en ella se facilita a los estudiantes los materiales necesarios para cursar la asignatura, siguiendo un cronograma que se determina a principio de curso. A través de esta plataforma se proponen las actividades que el alumno va realizando para poder hacer un seguimiento continuado de la materia, así como los controles para revisar los conocimientos adquiridos.

También nos sirve de apoyo a la hora de impartir docencia el webinar Elluminate, en el que podemos crear aulas virtuales para reunirnos con los estudiantes y llevar a cabo actividades de forma síncrona, utilizando los mismos instrumentos que en una clase tradicional y pudiendo, por ejemplo, proyectar presentaciones o realizar clases magistrales. A este respecto, como señala Gorka Palacio, "estamos viviendo ya la transformación de las aulas en verdaderos espacios de estudio con conectividad de banda ancha a Internet, para una enseñanza constructivista y conectivista en la que los estudiantes y el profesor estarán siempre en línea y tendrán gran parte de sus contenidos en aplicaciones Web 2.0." (Palacio, 2010: 97).

Además de estas herramientas básicas en la educación a distancia, para el módulo de radio hemos utilizado otras más específicas, como el programa Audacity, que nos sirve para capturar, editar y mezclar distintas pistas de sonido y obtener un archivo listo 
para emitir y algunos almacenamientos de archivos en línea para poder acceder a las grabaciones finales, así como la tecnología de Google Docs para realizar una encuesta a los estudiantes que nos permitiera conocer su satisfacción con la metodología empleada.

\section{Desarrollo}

Dentro de la asignatura de Comunicación Radiofónica, empleamos las primeras unidades en repasar los conceptos generales, la historia y las particularidades del medio radiofónico. Posteriormente, explicamos a los estudiantes las pautas para poder realizar grabaciones en sus propias casas y para instalar en sus ordenadores los programas que les permitan la grabación y edición de los archivos de audio.

La heterogeneidad de nuestros alumnos hace que muchos de ellos conozcan, de antemano, la forma de grabar y editar sonido y además, se abren múltiples posibilidades para hacerlo. Nos encontramos con que algunos de ellos trabajan o colaboran con emisoras de radio, locales o nacionales, y que es en los mismos estudios en los que se les permite elaborar sus prácticas y hacer grabaciones. En otros casos, los estudiantes disponen de micrófonos conectados a las tarjetas de sonido de sus ordenadores y finalmente, también podemos observar que algunos de ellos, fieles seguidores de las tecnologías más punteras, disponen de teléfonos de última generación o de tabletas que les permiten hacer grabaciones con una calidad superior a la esperada inicialmente.

Además, el hecho de que los programas de edición de sonido resulten bastante intuitivos y las herramientas básicas se puedan dominar con unas horas de práctica, hace que los alumnos se motiven para poder elaborar sus archivos y presentarlos como parte de sus actividades. Consideramos que esta asignatura, a pesar de tener un carácter más introductorio, debía incluir la realización de prácticas breves e individuales que familiaricen al estudiante con las técnicas y les permitan, posteriormente, desarrollar la actividad con mayor facilidad y empleando menos tiempo. Por ello, en las dos últimas unidades de la asignatura, planteamos la siguiente actividad: Elaborar una crónica para un boletín informativo, similar a las que realizan habitualmente en radio, de un minuto aproximadamente de duración, sobre una información elegida libremente por el estudiante, y en la que se recojan los conocimientos adquiridos anteriormente; haciendo especial hincapié en la correcta utilización del lenguaje y la redacción propia del medio radiofónico, la estructuración de la información, así como la "puesta en escena" de la misma. El ejercicio incluye escribir y registrar la grabación de la lectura de la crónica y enviarla a través de los buzones que a tal efecto existen en el Aula Virtual.

Tras comprobar que los estudiantes contaban con los medios para poder realizar la grabación sin dificultad y que el resultado de la misma era satisfactorio, pusimos en marcha una segunda actividad similar, variando únicamente la temática de la información, que ofreció resultados muy parecidos. Estas dos grabaciones de la asignatura de Comunicación Radiofónica habían familiarizado a los estudiantes con las destrezas necesarias para poder pasar a una segunda fase en la práctica de la radiodifusión y dar el salto desde los géneros aislados a los formatos más complejos. 
El rendimiento de los estudiantes, su disposición y la buena respuesta ofrecida por ellos hizo que planteáramos la asignatura de Periodismo Radiofónico de forma ambiciosa y que, tomando el manual como referencia, nos propusiéramos crear espacios radiofónicos completos, con las características de los formatos profesionales y que se pudieran elaborar de igual forma que se hace en los medios reales, salvando las distancias inevitables.

Para ello, antes de comenzar la asignatura, dividimos a los estudiantes en grupos de cinco ó seis integrantes con el fin de simular un equipo real de trabajo. Uno de ellos, sería el encargado de dirigir, editar y remitir el resultado final de su trabajo, un archivo de audio editado y listo para emitir, elaborado por todo el grupo. Este puesto, que simularía al del director, editor o presentador del espacio, debería ser rotatorio para que todos los integrantes del grupo pudieran en algún momento desempeñar este papel.

Tras esta división inicial, se indicó a los estudiantes cuál sería el patrón a seguir para realizar las distintas actividades en grupo, intentando seguir el mismo esquema de trabajo que se lleva a cabo en la redacción de una emisora de radio. Para ello, se estableció un sistema en cuatro pasos.

1. Reunión del grupo para decidir quién iba a ser el responsable de la edición del espacio a realizar, qué temas se iban a incluir en el mismo, qué duración deberían tener, con qué géneros se iba a trabajar y, finalmente, para hacer un reparto de la tarea entre los integrantes del grupo y fijar la hoja de ruta, con las fechas de entrega, de cada apartado.

2. Trabajo individual de los miembros del grupo para redactar, elaborar y enviar su parte del trabajo, siguiendo las instrucciones del editor de cada espacio.

3. Trabajo final del editor o director que consistía en la recopilación y edición del trabajo elaborado por los compañeros para unificarlo y poder presentar el formato solicitado en cada actividad y posteriormente, enviarlo al resto de los miembros del equipo para que dieran su visto bueno o aportaran las modificaciones que consideraran necesarias.

4. Envío de la actividad al profesor, mediante el buzón disponible en el Aula Virtual o bien, dependiendo del tamaño del archivo de audio resultante, a través de un servicio de transferencia de archivos.

Teniendo en cuenta estas premisas, y siguiendo las pautas teóricas que les ofrecía el manual de la asignatura, junto con el material aportado por el docente, los estudiantes debían elaborar los siguientes espacios: un boletín informativo, un programa deportivo, un programa musical, un guión dramático y un anuncio publicitario.

Para llevar a cabo estas actividades, los estudiantes podían solicitar al profesor el material y las herramientas que consideraran necesarias en cada momento. Una de las más utilizadas fueron las salas de Elluminate, que se creaban para que los estudiantes pudieran hacer allí, de forma síncrona, sus "reuniones de redacción".

Durante el desarrollo de las actividades, también se produjo un contacto continuado entre el docente y los alumnos para solventar, por ejemplo, dudas acerca del formato en el que se podían enviar los archivos o la mejor forma de hacerlos llegar; bien a través de plataformas virtuales que permitieran un almacenamiento mayor que los 
correos electrónicos tradicionales, bien descomponiendo los archivos de sonido en varias partes consecutivas que se pudieran enviar por correo electrónico, etc.

Desde el punto de vista del docente, el desarrollo de las actividades siguió el ritmo necesario y la calidad de las mismas fue, en la mayor parte de los casos, muy satisfactoria e incluso mejor de la esperada inicialmente. La implicación de los estudiantes alcanzó cotas muy significativas y prácticamente ninguno de ellos representó problemas para el resto del grupo. En conjunto, todos ellos buscaron sonidos y músicas para elaborar sus propias caretas, cortinillas, indicativos, etc., y obtuvieron resultados bastante profesionales.

\subsection{Valoración de las herramientas metodológicas empleadas por parte del alum- nado}

Al finalizar el curso se propuso a los estudiantes una encuesta anónima para que valoraran y criticaran el desarrollo de la asignatura de Periodismo Radiofónico. Su valoración del módulo de radio y en especial de la parte práctica de los mismos ha sido positiva, en líneas generales, y destacan el incremento de la relación con sus compañeros y la simulación de la actividad real del periodista de radio como los aspectos más reseñables.

La encuesta estaba estructurada en tres apartados: el primero de ellos en el que el estudiante debía elegir una puntuación en una escala del uno al cinco, según su grado de acuerdo y aceptación de la idea propuesta; la segunda en la que debía elegir entre varias opciones para determinar el tipo de herramientas que había utilizado y una tercera parte de respuestas libres para poder incluir las recomendaciones y opiniones personales del alumno.

De los resultados obtenidos podemos deducir, por ejemplo, que ocho de cada diez estudiantes está de acuerdo o muy de acuerdo en que las actividades destinadas a simular el trabajo real de una redacción de radio son útiles para su aprendizaje, mientras un porcentaje inferior al 10\% asegura que no está nada de acuerdo con esta afirmación. Asimismo, el conjunto de estudiantes que considera que este tipo de ejercicios resultan muy útiles supera el $80 \%$ del total de los alumnos que completaron la encuesta.

Respecto a la utilidad, a efectos de socialización y de mejora de la relación entre los compañeros de clase que ha tenido el desarrollo de este tipo de actividades, en torno al $80 \%$ consideran que ha sido útil. Sobre las herramientas más utilizadas para el desarrollo de las actividades de la asignatura, la mayor parte de los estudiantes asegura haberse comunicado con sus compañeros a través del correo electrónico y Elluminate, mientras que en menor proporción, algunos también han utilizado el teléfono $\mathrm{u}$ otros sistemas.

En cuanto a la elaboración y el desarrollo de la actividad, la proliferación de dispositivos electrónicos con aplicaciones capaces de registrar el sonido con una calidad más que aceptable hace que el porcentaje de alumnos que utilizó dispositivos móviles como teléfonos, smartphones, tabletas y similares supere el $40 \%$; mientras que un porcentaje parecido empleó la grabadora tradicional, independientemente del formato de la misma, y menos del 10\% recurrió a los micrófonos integrados en ordenadores portátiles o de sobremesa. 
A la hora de editar el sonido para obtener la mejor calidad y el resultado final de las actividades, uno de cada tres estudiantes eligió Audacity, el software recomendado por la asignatura, y dada la experiencia de algunos de los estudiantes, estos se decantaron por el empleo de otros programas informáticos, entre ellos el SoundForge.

Finalmente, a modo de resumen sobre las preguntas de respuesta libre incluidas en la encuesta, podemos destacar que la mayor parte de los estudiantes se ha mostrado satisfecho con las actividades y cree que ayudan a acercar la realidad del periodismo radiofónico a las aulas. Consideran que existen ciertas dificultades a la hora de instalar algunos programas informáticos útiles para grabar y editar sonido pero reconocen que han podido solventar estos problemas con otro tipo de software y proponen que se amplíe más la formación práctica en cuanto a locución y vocalización, con el fin de mejorar la puesta en escena de las actividades.

\section{Conclusiones}

La sociedad y los medios de comunicación viven una época de constante cambio y transformación, un hecho que hace necesarios nuevos enfoques educativos para la formación de profesionales de nuestro tiempo. La enseñanza meramente teórica continúa siendo básica para los nuevos periodistas pero si no se les dota de las herramientas prácticas necesarias para ejercer su oficio de forma adecuada, máxime en una disciplina como es la radiodifusión, en la que el entrenamiento adecuado es fundamental para el desarrollo de las tareas específicas que el medio radiofónico exige.

Por otra parte, mediante el desarrollo de actividades prácticas, creadas por los estudiantes, se puede estimular a los participantes para que adquieran y practiquen ciertas habilidades que no han estado especialmente contempladas en los métodos tradicionales. "La radio fomenta el desarrollo de las capacidades imaginativa de los estudiantes, al tiempo que se revela como una herramienta transversal que puede favorecer el aprendizaje de otras materias" (Perona y Barbeito, 2007: 35).

La plataforma Moodle se ha revelado como un medio excelente para poder llevar la teoría de las asignaturas relacionadas con la radiodifusión desde el campo teórico hasta su aplicación práctica, por parte de los estudiantes. Una revelación de especial importancia para la UDIMA, dado que las enseñanzas del Grado de Periodismo se imparten a distancia, con la dificultad añadida que se presuponía inicialmente.

En este mismo sentido, la predisposición de la sociedad hacia las herramientas tecnológicas y la familiarización de los estudiantes con ellas hace que la educación a distancia pueda sacar provecho de ellas para solventar las dificultades que por sí misma puede presentar a la hora de realizar tareas prácticas y de grupo.

En cuanto a la percepción sobre la realización de este tipo de actividades, el profesorado que imparte las asignaturas del módulo de radio de la UDIMA ha podido constatar el elevado nivel de participación y de implicación en las actividades prácticas.

De la misma manera, la encuesta realizada para conocer el grado de satisfacción de los estudiantes con la realización de prácticas a distancia, ha arrojado resultados muy positivos; destacando especialmente el incremento de la interacción entre compañeros y la motivación que supone para un estudiante de periodismo la simulación del trabajo real de la redacción de una emisora de radio. 
A la vista de los resultados de esta experiencia, podemos afirmar que se impone la necesidad de un cambio de enfoque en la enseñanza de las asignaturas de radio de las universidades, de acuerdo con los principios del Espacio Europeo de Educación Superior (EEES) y con las exigencias que plantea la realidad práctica de los medios de comunicación actuales. Una modificación en las enseñanzas que puede dar resultados muy satisfactorios, aún en el entorno complejo de la educación a distancia, y que prepara a los estudiantes para poder afrontar los desafíos futuros de la profesión.

\section{Referencias bibliográficas}

AGENCIA NACIONAL DE EVALUACIÓN DE CALIDAD Y ACREDITACIÓN (ANECA) (2005): Libro Blanco. Títulos de Grado en comunicación. Disponible en http://www.aneca.es/var/media/150336/libroblanco_comunicacion_def.pdf [Consultado: 20 de septiembre de 2012]

MERAYO PÉREZ, Arturo (2010): Didáctica de la Comunicación Radiofónica. Disponible en http://www.bocc.ubi.pt/pag/merayo-arturo-didactica-comunication-radiofonica.pdf. [Consultado: 12 de agosto de 2012].

PALACIO, Gorka J. (2010): "Sistemas con contenido audiovisual y multimedia para el desarrollo de entornos de e-learning 2.0". En LANDETA, A. (coord.): Nuevas tendencias de e-learning y actividades didácticas innovadoras. Madrid, CEF.

PERONA PÁEZ, Juan José y BARBEITO VELOSO, Mariluz (2007): Modalidades educativas de la radio en la era digital, en Revista Icono $14, \mathrm{n}^{\circ}$ 9. Junio.

TENORIO SANTOS, Iván (2008): La nueva radio. Manual completo del radiofonista moderno. Barcelona. Editorial Marcombo.

\section{Yolanda BERDASCO GANCEDO}

Universidad a Distancia de Madrid (UDIMA)

Profesora en el Departamento de Periodismo, Historia y Humanidades.

yolanda.berdasco@udima.es

\section{Ana Virginia RUBIO JORDÁN}

Universidad a Distancia de Madrid (UDIMA)

Profesora en el Departamento de Periodismo, Historia y Humanidades.

anavirginia.rubio@udima.es 